\title{
Superior Semicircular Canal Dehiscence Syndrome - Diagnosis and Surgical Management
}

\author{
Marite Palma Diaz ${ }^{1,2}$ Juan Carlos Cisneros Lesser ${ }^{3}$ \\ 1 Otorhinolaryngology and Neurotology Department, Instituto \\ Nacional de Neurología y Neurocirugía Dr. Manuel Velasco Suárez, \\ México, DF, Mexico \\ 2 Neurology Center, Centro Médico American British Cowdray, \\ México, DF, Mexico \\ 3 Otorhinolaryngology and Neurotology Depaertment, Instituto \\ Nacional de Rehabilitación, México, DF, Mexico
}

Alfredo Vega Alarcón ${ }^{1,2}$

Int Arch Otorhinolaryngol 2017;21:195-198.

\begin{abstract}
Keywords

- superior semicircular canal

- superior semicircular canal dehiscence

- vertigo

- middle cranial fossa

Introduction Superior semicircular canal dehiscence syndrome was described by Minor et al in 1998. It is a troublesome syndrome that results in vertigo and oscillopsia induced by loud sounds or changes in the pressure of the external auditory canal or middle ear. Patients may present with autophony, hyperacusis, pulsatile tinnitus and hearing loss. When symptoms are mild, they are usually managed conservatively, but surgical intervention may be needed for patients with debilitating symptoms.

Objective The aim of this manuscript is to review the different surgical techniques used to repair the superior semicircular canal dehiscence.

Data Sources PubMed and Ovid-SP databases.

Data Synthesis The different approaches are described and discussed, as well as their limitations. We also review the advantages and disadvantages of the plugging, capping and resurfacing techniques to repair the dehiscence.

Conclusions Each of the surgical approaches has advantages and disadvantages. The middle fossa approach gives a better view of the dehiscence, but comes with a higher morbidity than the transmastoid approach. Endoscopic assistance may be advantageous during the middle cranial fossa approach for better visualization. The plugging and capping techniques are associated with higher success rates than resurfacing, with no added risk of hearing loss.
\end{abstract}

Address for correspondence Marite Palma Diaz, MD, Insurgentes Sur 3877, Tlalpan. Mexico City, Mexico (e-mail: maritepalma@hotmail.com).

\section{Introduction}

The superior semicircular canal dehiscence (SSCD) syndrome was described by Lloyd B. Minor et $\mathrm{al}^{1}$ in 1998 . This syndrome is characterized by the formation of a "third opening" or "third window" between the superior semicircular canal and the middle cranial fossa, secondary to a bony defect in the canal. This abnormal communication can result in vertigo and oscillopsia induced by loud sounds (Tullio's phenomenon), by Valsalva maneuvers, or by changes in pressure in the external auditory canal (Hennebert's sign). The patients may also present autophony, hyperacusis, pulsatile tinnitus and hearing loss. In patients with debilitating symptoms and low health utility scores, a surgical management may be offered, since the manifestations of the disease and the utility score may improve with surgery. The different received

December 2, 2016

accepted

February 1, 2017
DOI http://dx.doi.org/ 10.1055/s-0037-1599785. ISSN $1809-9777$.
Copyright (e 2017 by Thieme-Revinter

Publicações Ltda, Rio de Janeiro, Brazil

\section{License terms}

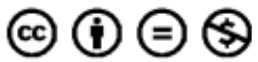


surgical techniques used to approach and repair the SSCD include the middle cranial fossa approach, the transmastoid approach, the endoscopic-assisted middle cranial fossa approach, and the round window tissue reinforcement. In this review of the literature we analyze this clinical syndrome and its diagnosis, as well as the aforementioned surgical approaches and techniques performed to repair the dehiscence.

\section{Review of the Literature and Discussion}

There are two functional windows in the cochleovestibular system, the oval window, which allows the passage of soundwaves into the inner ear's scala vestibuli through the stapes footplate, and the round window, involved in the release of sound and mechanical energy from de scala tympani out of the inner ear. This is a hydraulically closed system. When a dehiscence in the superior semicircular canal is created, the hydro-acoustic waves flowing through the cochlea are inadvertently transmitted throughout the labyrinthine system. This causes the activation of the vestibular system, and thus vertigo is perceived. In addition, the intracranial pressure may be transmitted through the dehiscent superior canal, also causing the stimulation of vestibular end organs until the round window releases this pressure. ${ }^{2}$ In a more detailed manner, a SSCD alters the hydrodynamic stability of this system, enabling an exaggerated movement of the endolymph. Since a third window is present, the transmission of pressure through the inner ear may result in an outward bulging of the membranous canal at the bony defect to the side of the middle cranial fossa. Hydraulically speaking, it would serve as a pressure release valve and, therefore, less pressure would be distributed in the inner ear, as it would in a closed hydraulic system. ${ }^{3}$ When this outward bulging of the membranous labyrinth is produced, the disturbed endolymph fluid motion causes the ampullofugal deflection of the cupula of the superior semicircular canal, which the brain perceives as body movement, thus resulting in vestibular symptoms. ${ }^{4}$ Intracranial pressure fluctuations or negative pressure in the external or middle ear may lead to the contrary, an abnormal reversal of energy through the dehiscence into the labyrinth, resulting in an inward bulging of the membranous canal with a consequent ampullopetal deflection of the cupula. ${ }^{3,4}$ These theories support the noise or pressure-induced vertigo observed in the SSCD syndrome.

The increased compliance of the inner ear explained earlier also contributes to the conductive hearing loss and the perception of the pulsatile tinnitus observed in these patients. $^{2}$ The pathologic opening from the SSCD may weaken the energy transmission produced by the movement of the stapes footplate, resulting in the reduction of sound transmission to the cochlea and hearing loss. On the other hand, the normal impedance inequality of the oval and round windows may be altered by the third window, which results in bone hyperconduction that the patient perceives as autophony or hyperacusis. ${ }^{5}$

The diagnosis is suspected by the clinical manifestations mentioned earlier. Nystagmus evoked by sound or pressure, with eye movements oriented in the same plane as the dehiscent semicircular canal, is a classic feature. Audiometric findings typically include supernormal bone thresholds and a low-frequency conductive hearing loss. The tympanometric examination and acoustic reflexes are normal. ${ }^{6}$ Cervical vestibular evoked myogenic potential (cVEMP) testing in patients with SSCD syndrome will often have responses with lower thresholds (less than 70 decibels [dB] to tone burst testing) than in people without the syndrome. ${ }^{7}$ In 2012, Zuniga et al ${ }^{8}$ showed that cVEMP threshold results showed a sensitivity and a specificity ranging from $80 \%$ to $100 \%$ for the diagnosis of SSCD syndrome. In contrast, the ocular cVEMP amplitudes demonstrated a sensitivity and a specificity greater than $90 \%$. Finally, when a SSCD syndrome is suspected, a temporal bone computed tomography (CT) scan confirms the diagnosis. The best images are seen in a high resolution $0.5 \mathrm{~mm}$ collimation, and projections in the plane of the superior canal (Pöschl views) and in a perpendicular plane to this same canal (Stenvers views). ${ }^{9}$ Two types of SSCD have been described after a CT examination: dehiscence of the arcuate eminence or dehiscence in the region of the superior petrosal sinus. Studies have found that magnetic resonance imaging (MRI) is also very useful for diagnosing SSCD syndrome. In 2013, Browaeys et $\mathrm{al}^{10}$ described that when diagnosing SSCD syndrome, the MRI had a sensitivity of $100 \%$, a specificity of $96.5 \%$, a positive predictive value of $61.1 \%$, and a negative predictive value of $100 \%$ in comparison with the CT.

The etiology is unknown. The first manifestations of the syndrome usually occur during adulthood; nevertheless, a congenital anomaly is suspected, since thinning of the bone overlying the superior semicircular canal occurs bilaterally in almost one-third of the patients, and there are some cases that began having symptoms during childhood. ${ }^{11,12}$ In contrast to adults, children with SSCD syndrome usually present with auditory symptoms first, and conservative management should be carried out as often as possible. ${ }^{12}$

Another possibility for the pathophysiology of this disease is that it may be an acquired condition. Repeated, low intensity cranial trauma from combat sports or diving, for instance, has been related to this disease. A defect in the floor of the middle cranial fossa, at the level of the canal, may also occur secondary to increments in the pressure of the cerebrospinal fluid. ${ }^{11}$

Patients with mild symptoms should be treated with conservatively. For patients with debilitating symptoms and lower health utility scores, surgical management may be offered. Both the clinical manifestations of the disease and the so-called health utility scores improve after surgery. ${ }^{13}$ When surgery is necessary, the bony dehiscence can be resurfaced, plugged or capped by different surgical approaches. ${ }^{14-18}$

\section{Middle Cranial Fossa Approach}

This approach to treat SSCD syndrome was first described by Minor et al. ${ }^{14} \mathrm{~A} 4 \times 4 \mathrm{~cm}$ craniotomy is drilled. The temporal lobe is retracted, and the arcuate eminence is identified. At this point, the dehiscence may be visualized. The canal is opened using a diamond drill, and then it is plugged. The 
canal may also be capped or resurfaced with bone pate, bone wax, hydroxyapatite cement or soft tissue.

The advantages of the middle fossa craniotomy include the direct access to the arcuate eminence defect without the need for labyrinthine bone removal and the exposure of the surrounding cranial base if the repair is needed for other associated tegmen defects.

Concomitant resurfacing of the tegmen mastoideum and tympani is performed, since the floor of the middle cranial fossa is usually thin. Resurfacing of the dehiscent canal also prevents chronic stimulation from the pulsating temporal lobe.

\section{Transmastoid Approach}

The surgical technique begins with a post auricular incision, as it usually performed for a mastoidectomy. Supra and subperiosteal flaps are made, and then a mastoidectomy is performed using a cutting burr with suction irrigation. The sigmoid sinus, the posterior and middle fossa dura, and the pre-sigmoid area are all skeletonized. The horizontal, posterior and superior semicircular canals are identified and skeletonized with a diamond burr. The area of dehiscence is identified, and the middle fossa dura is carefully elevated from de dehiscent superior semicircular canal. In patients with dehiscence at the superior petrosal sinus, the sinus was exposed at the sinodural angle, posterior to the solid angle, and followed to the superior canal. ${ }^{16}$ Two points of the bony labyrinth are fenestrated with a $1 \mathrm{~mm}$ diamond burr, and the endosteum is opened just inferior to the fenestrated apex of the superior canal, on the ampullated and non-ampullated portions of the canal. ${ }^{19}$ Care is taken to avoid suction or manipulation of the membranous labyrinth to prevent hearing loss or chronic disequilibrium after surgery. ${ }^{20}$ Bone dust, bone wax, bone pate, fascia and even bone chips may be used to fill the lumen of the superior canal at the points of fenestration (plugging the canal). If bone wax is used, Cheng et $\mathrm{al}^{21}$ recommend that the applications of two wax spheres, $2 \mathrm{~mm}$ in size, is sufficient to occlude the canal without risk of damaging the neuroepithelium, which prevents vestibular hypofunction and hearing loss after the repair. Conchal cartilage is harvested and placed in an intracranial extradural position, repairing the middle cranial fossa floor defect, and finally the wound is closed in a regular fashion. ${ }^{19}$

When the transmastoid middle fossa resurfacing is selected, after dissecting the middle cranial fossa dura from the dehiscent canal, tragal perichondrium is placed in the space between the dura and the dehiscence by folding it over the top of an annulus elevator and inserting it over the superior canal. The dura and overlying temporal lobe or superior petrosal sinus will stabilize the position of the graft, with no tendency to extrude. ${ }^{16}$

It is well known that the transmastoid approach avoids the risks of performing a craniotomy in the middle fossa, resulting in lower morbidity and a shorter hospital stay. The disadvantage of transmastoid procedures is that the dehiscence is visualized with more difficulty compared to the middle fossa approach. ${ }^{22}$ Another limitation is when the tegmen hangs too low, precluding the safe exposure and manipulation of the dura just lateral to the superior semicircular canal. A preoperative CT scan is very important in order to anticipate this difficulty, and it could precisely identify the size, extension and location of the dehiscence.

\section{Endoscopic-assisted Middle Cranial Fossa Repair}

The endoscopic-assisted middle fossa procedure appears to be advantageous when compared with binocular microscopy, since it provides a high definition view of the middle cranial fossa. In this surgical technique, following the incision, a temporalis fascia graft is harvested. A periosteal flap is made, and a minicraniotomy $(3 \times 2 \mathrm{~cm})$ is performed. Under microscopy, the dura is gently elevated off the tegmen mastoideum and tegmen tympani until the arcuate eminence is visualized. Then, a $3 \mathrm{~mm}$ wide, $14 \mathrm{~cm}$ long, $30^{\circ}$ rigid endoscope is introduced in order to visualize the defect. The House-Urban middle fossa retractor is used to maintain the retraction of the temporal lobe while working with the endoscope. The dura is dissected from the medial aspect of the defect under endoscopic view. At this point, a gentle occlusion of the ampullated and non-ampullated limbs of the defect is performed using bone wax. ${ }^{15}$ Peng et $\mathrm{al}^{23}$ reported 10 cases managed with the endoscopic-assisted middle fossa technique in which they used hydroxyapatite bone cement to resurface the superior canal with good results. In 2014, Carter et $\mathrm{al}^{18}$ reported their experience with 5 patients managed endoscopically. They mentioned that endoscopy enhances the visualization of the superior canal defect, allows for transillumination, and reduces temporal lobe retraction, making it a useful adjunct in craniotomies used to repair SSCD defects.

\section{Round Window Reinforcement}

This surgical technique is performed using a traditional tympanomeatal flap approach under general or local anesthesia. If necessary, a drill or curettage is used to enlarge the posterior auditory canal wall in order to gain adequate exposure of the round window niche. This niche and the promontory are denuded of mucosa, and the round window is reinforced with temporalis fascia, tragal cartilage, perichondrium, fat or connective tissue. ${ }^{24}$ The tympanomeatal flap is repositioned and the external auditory canal is packed.

The round window reinforcement is a procedure with low risk compared to the middle fossa or transmastoid approaches, and it may be offered as a first procedure in patients with mild symptoms. Complete occlusion of the round window is another technique that has been used for SSCD syndrome, but some authors suggest that it should not be recommended, since it has been noticed that symptoms may become worse in the late postoperative period. ${ }^{24}$

\section{Plugging or Capping versus Resurfacing}

Different studies have shown that the combination of plugging and resurfacing achieves better long-term control of the symptoms than resurfacing alone. When only resurfacing is performed, a complete sealing of the defect is not guaranteed, and this area may remain sensitive to pressure changes. $^{25,26}$ Goddard and Wilkinson, ${ }^{22}$ in 2014, showed 
excellent hearing outcomes and a reduction of the preoperative symptoms in 24 ears in which the plugging technique was used.

A meta-analysis of 64 surgical procedures for SSCD syndrome indicated that capping and plugging had a significantly higher success rate when compared to resurfacing. ${ }^{27}$

\section{Final Comments}

Even though the conservative management, which avoids the precipitating stimuli and vestibular rehabilitation, is most often indicated, patients with debilitating symptoms may be offered surgical repair of the SSCD. Each of the surgical approaches has advantages and disadvantages. The middle fossa approach gives a better view of the dehiscence, which may prove beneficial at the moment of the repair, but comes with a higher morbidity than the transmastoid route. Endoscopic assistance may also improve the middle cranial fossa approach, giving it an advantage. The plugging and capping techniques are associated with higher success rates than resurfacing, with no added risk of hearing loss; thus, those techniques should be recommended.

Conflict of Interest

The authors declare no conflicts of interest.

\section{References}

1 Minor LB, Solomon D, Zinreich JS, Zee DS. Sound- and/or pressureinduced vertigo due to bone dehiscence of the superior semicircular canal. Arch Otolaryngol Head Neck Surg 1998;124(03): 249-258

2 Minor LB. Clinical manifestations of superior semicircular canal dehiscence. Laryngoscope 2005;115(10):1717-1727

3 Rajan GP, Leaper MR, Goggin L, Atlas MD, Boeddinghaus R, Eikelboom RK. The effects of superior semicircular canal dehiscence on the labyrinth: does size matter? Otol Neurotol 2008; 29(07):972-975

4 Banerjee A, Whyte A, Atlas MD. Superior canal dehiscence: review of a new condition. Clin Otolaryngol 2005;30(01):9-15

5 Cremer PD, Minor LB, Carey JP, Della Santina CC. Eye movements in patients with superior canal dehiscence syndrome align with the abnormal canal. Neurology 2000;55(12):1833-1841

6 Castellucci A, Brandolini C, Piras G, Modugno GC. Tympanometric findings in superior semicircular canal dehiscence syndrome. Acta Otorhinolaryngol Ital 2013;33(02):112-120

7 Niesten ME, McKenna MJ, Herrmann BS, Grolman W, Lee DJ. Utility of cVEMPs in bilateral superior canal dehiscence syndrome. Laryngoscope 2013;123(01):226-232

8 Zuniga MG, Janky KL, Nguyen KD, Welgampola MS, Carey JP. Ocular versus cervical VEMPs in the diagnosis of superior semicircular canal dehiscence syndrome. Otol Neurotol 2013;34(01):121-126
9 Branstetter BF IV, Harrigal C, Escott EJ, Hirsch BE. Superior semicircular canal dehiscence: oblique reformatted CT images for diagnosis. Radiology 2006;238(03):938-942

10 Browaeys P, Larson TL, Wong ML, Patel U. Can MRI replace CT in evaluating semicircular canal dehiscence? AJNR Am J Neuroradiol 2013;34(07):1421-1427

11 Hegemann SC, Carey JP. Is superior canal dehiscence congenital or acquired? A case report and review of the literature. Otolaryngol Clin North Am 2011;44(02):377-382, ix

12 Lee GS, Zhou G, Poe D, et al. Clinical experience in diagnosis and management of superior semicircular canal dehiscence in children. Laryngoscope 2011;121(10):2256-2261

13 Remenschneider AK, Owoc M, Kozin ED, McKenna MJ, Lee DJ, Jung DH. Health Utility Improves After Surgery for Superior Canal Dehiscence Syndrome. Otol Neurotol 2015;36(10):1695-1701

14 Chien WW, Carey JP, Minor LB. Canal dehiscence. Curr Opin Neurol 2011;24(01):25-31

15 Shaia WT, Diaz RC. Evolution in surgical management of superior canal dehiscence syndrome. Curr Opin Otolaryngol Head Neck Surg 2013;21(05):497-502

16 Teixido M, Seymour PE, Kung B, Sabra O. Transmastoid middle fossa craniotomy repair of superior semicircular canal dehiscence using a soft tissue graft. Otol Neurotol 2011;32(05):877-881

17 Amoodi HA, Makki FM, McNeil M, Bance M. Transmastoid resurfacing of superior semicircular canal dehiscence. Laryngoscope 2011;121(05):1117-1123

18 Carter MS, Lookabaugh S, Lee DJ. Endoscopic-assisted repair of superior canal dehiscence syndrome. Laryngoscope 2014;124(06):1464-1468

19 Deschenes GR, Hsu DP, Megerian CA. Outpatient repair of superior semicircular canal dehiscence via the transmastoid approach. Laryngoscope 2009;119(09):1765-1769

20 Niesten ME, McKenna MJ, Grolman W, Lee DJ. Clinical factors associated with prolonged recovery after superior canal dehiscence surgery. Otol Neurotol 2012;33(05):824-831

21 Cheng YS, Kozin ED, Remenschneider AK, Nakajima HH, Lee DJ. Characteristics of wax occlusion in the surgical repair of superior canal dehiscence in human temporal bone specimens. Otol Neurotol 2016;37(01):83-88

22 Goddard JC, Wilkinson EP. Outcomes following semicircular canal plugging. Otolaryngol Head Neck Surg 2014;151(03):478-483

23 Peng A, Shaia WT. Endoscopic repair of superior canal dehiscence. Presented at the Virginia Society of Otolaryngology Annual Meeting: Charlottesville, Virginia, May 1-2:2010

24 Silverstein H, Kartush JM, Parnes LS, et al. Round window reinforcement for superior semicircular canal dehiscence: a retrospective multi-center case series. Am J Otolaryngol 2014;35(03): 286-293

25 Ward BK, Agrawal Y, Nguyen E, et al. Hearing outcomes after surgical plugging of the superior semicircular canal by a middle cranial fossa approach. Otol Neurotol 2012;33(08):1386-1391

26 Fiorino F, Barbieri F, Pizzini FB, Beltramello A. A dehiscent superior semicircular canal may be plugged and resurfaced via the transmastoid route. Otol Neurotol 2010;31(01):136-139

27 Vlastarakos PV, Proikas K, Tavoulari E, Kikidis D, Maragoudakis P, Nikolopoulos TP. Efficacy assessment and complications of surgical management for superior semicircular canal dehiscence: a meta-analysis of published interventional studies. Eur Arch Otorhinolaryngol 2009;266(02):177-186 